\title{
The Predictive Value of the
}

\section{Monocyte-to-Lymphocyte Ratio and}

\section{Monocyte-to-Haematocrit Ratio for Cardiac Rupture Patients with Acute Myocardial Infarction: A Propensity Score Matching Analysis}

\author{
Kai Dai $\mathbb{D}^{1, *}$, Zhibing $\mathrm{Li}^{2, *}$, Yafei Luo',*, Qianhui Xiong ${ }^{1, *}$, Yao Xiong ${ }^{3, *}$, Zhifang Song ${ }^{1}, *$, Wenjun Xiong ${ }^{1}$ \\ 'Department of Cardiovascular Medicine, The First Affiliated Hospital of Nanchang University, Medical Department of Nanchang University, Nanchang, 330006, \\ Jiangxi, People's Republic of China; ${ }^{2}$ Department of Intensive Care Unit, The First Affiliated Hospital of Nanchang University, Medical Department of Nanchang \\ University, Nanchang, 330006, Jiangxi, People's Republic of China; ${ }^{3}$ Department of Cardiovascular Medicine, Jiangxi Provincial People's Hospital Affiliated to \\ Nanchang University, Medical Department of Nanchang University, Nanchang, 330006, Jiangxi, People's Republic of China
}

Correspondence: Wenjun Xiong, Department of Cardiovascular Medicine, The First Affiliated Hospital of Nanchang University, Medical Department of Nanchang University, No. 46 I Bayi Avenue, Nanchang, 330006, Jiangxi, People’s Republic of China, Tel +86I3767970074, Email xiongwenjunnc@foxmail.com

*These authors contributed equally to this work

Background: Cardiac rupture (CR) is a serious complication of acute myocardial infarction (AMI). We aimed to explore the predictive value of blood cell parameters for identifying CR in patients with AMI using the introduction of propensity score matching (PSM).

Methods: This retrospective study enrolled patients who were diagnosed with AMI from January 2013 to May 2020. A total of 109 patients with CR were included, and 327 hospitalized non-CR patients were randomly selected at a 1:3 ratio. Based on the 1:1 nearest neighbour matching method by using SPSS, the covariances of the two groups were balanced. After PSM, the independent risk factors for CR were selected by using multivariate logistic regression analysis, and receiver operating characteristic (ROC) curve analysis was applied to evaluate the predictive value of blood cell parameters for CR. Ninety cases were matched successfully in each of the two groups.

Results: Among the 180 patients with AMI after PSM, the results of multivariate logistic regression analysis showed that the monocyte-tolymphocyte ratio (MLR) $(\mathrm{OR}=3.57,95 \% \mathrm{CI}: 1.28-9.97, \mathrm{P}=0.015)$ and monocyte-to-haematocrit ratio $(\mathrm{MHR})(\mathrm{OR}=1.80,95 \% \mathrm{CI}: 1.02-3.20$, $\mathrm{P}=0.043$ ) were independently related to the risk of CR. Additionally, the MLR (area under the curve (AUC): 0.74) and MHR (AUC: 0.73 ) were useful for distinguishing CR patients after PSM. To differentiate CR patients from the control subjects, the optimal cut-offs of the MLR and MHR were 0.61 (63\% sensitivity and $80 \%$ specificity) and 2.06 (57\% sensitivity and $81 \%$ specificity), respectively.

Conclusion: The blood cell parameters MLR and MHR were independently correlated with CR. Additional, the MLR and MHR were useful to predict $\mathrm{CR}$ in patients with AMI.

Keywords: cardiac rupture, acute myocardial infarction, blood cell parameters, monocyte-to-lymphocyte ratio, inflammatory, monocyte-to-haematocrit ratio

\section{Introduction}

With the application of timely reperfusion therapy in patients with acute myocardial infarction (AMI), hospital mortality has decreased significantly. However, cardiac rupture (CR) is still one of the main causes of death in patients with AMI. ${ }^{1,2} \mathrm{CR}$ is characterized by sudden onset and rapid progression. ${ }^{3}$ Therefore, it is of great clinical significance to identify patients with high-risk cardiac rupture early to actively prevent the occurrence of cardiac rupture and improve the prognosis of the patients. ${ }^{4}$ 
At present, the diagnosis of cardiac rupture is mainly based on clinical symptoms, signs, a combination of Doppler echocardiography and pericardiocentesis, and a lack of early predictive indicators. ${ }^{5}$ Blood cell analysis is a straightforward routine inspection method, and it is also the examination method with the highest utilization rate in clinical work. In recent years, many clinical studies have explored the relationships between various indicators of blood cell parameters and myocardial infarction. ${ }^{6,7}$ However, there is still a lack of research on the relationship between blood cell parameters and CR.

This study aimed to explore the relationship between these various indicators of blood cell parameters at admission and CR and identify the factors that can predict CR in the early stage.

\section{Methods}

\section{Study Patients and Design}

A retrospective collection of data from patients with AMI that were diagnosed at and admitted to the First Affiliated Hospital of Nanchang University and Jiangxi Provincial People's Hospital Affiliated to Nanchang University from January 2013 to May 2020 were divided into a CR group and a non-CR group. In this study, 109 patients with CR were included, and 327 hospitalized non-CR patients were randomly selected at a 1:3 ratio.

The inclusion criteria were as follows: (1) meeting the diagnostic criteria for AMI according to the definition of ACC/AHA; ${ }^{8}$ and (2) fitting the diagnosis of cardiac rupture (CR), ${ }^{9}$ which is confirmed by two professional cardiologists based on clinical symptoms and Doppler echocardiography or pericardial puncture. The exclusion criteria were as follows: (1) not diagnosed with CR due to AMI; (2) diagnosed with severe infection, liver and kidney disease, malignant tumours and haematological diseases; and (3) without complete clinical data.

\section{Data Collection}

The general and hospitalization data of patients who met the requirements of the inclusion criteria were collected. General information included sex, age, previous diabetes, hypertension, stroke, smoking, alcohol consumption and other medical history. Hospitalization data consisted primarily of vital signs, drug use, coronary artery stenosis status, coronary revascularization and routine blood results for the first time after admission. The related indicators of blood cell parameters included white blood cell count (WBC), red blood cell count (RBC), haemoglobin (HGB), haematocrit (HCT), mean corpuscular volume (MCV), mean corpuscular haemoglobin $(\mathrm{MCH})$, mean corpuscular haemoglobin concentration (MCHC), red blood cell distribution width (RDW), platelet count (PLT), mean platelet volume (MPV), plateletcrit (PCT), platelet distribution width (PDW), lymphocyte count (LY), monocyte count (MO), neutrophil count (NE), eosinophil count (EO), basophil count (BA), monocyte-to-lymphocyte ratio (MLR), monocyte-to-haematocrit ratio (MHR), and neutrophil-to-lymphocyte ratio (NLR).

The clinical symptoms of AMI manifest as severe chest pain with troponin elevation, new ischaemic changes on electrocardiogram, abnormal wall motion on Doppler echocardiography, and coronary angiography indicating the existence of thrombus. ${ }^{8}$ The diagnosis of CR was as follows: FWR was defined as the appearance of symptoms and signs of pericardial tamponade, electromechanical separation, a large amount of pericardial effusion and pericardial puncture with noncoagulable fluid; VSR was treated as a rough total systolic murmur with systolic tremor heard in the 4 to 5 intercostal spaces on the left edge of the sternum and a blood stream across the interventricular septum seen on echocardiography; PMR was based on a loud, rough systolic murmur heard at the apex of the heart and a large amount of mitral regurgitation or direct observation of papillary muscle rupture shown on echocardiography. Hypertension was described as systolic blood pressure (SBP) $>140 \mathrm{mmHg}$, diastolic blood pressure (DBP) $>90 \mathrm{mmHg}$ without antihypertensive drugs, and/or the use of antihypertensive drugs. ${ }^{9}$ Diabetes mellitus was defined as fasting blood glucose over $7.0 \mathrm{mmol} / \mathrm{L}$ and/or the use of antidiabetic drugs. ${ }^{10}$ Previous stroke was described as clinical symptoms of a neurological impairment lasting more than 24 hours or a new infarct lesion detected by computed tomography or head magnetic resonance imaging. ${ }^{11}$

\section{Statistical Analysis}

Data analysis of continuous and dichotomous variables was performed using SPSS software (version 25.0 for Windows, SPSS, Inc., Chicago, Illinois). The continuous variables in accordance with the normal distribution and the homogeneity 
of variance were expressed as the mean \pm standard deviation, and the $T$ test was applied for comparisons between the two groups. Otherwise, they were presented as median (interquartile range), and Mann-Whitney $U$-test was used for these continuous variables. Dichotomous variables are shown as frequencies (\%) and were tested by $\chi^{2}$-test or Fisher exact test if necessary. The potential risk factors for $\mathrm{CR}$ were analysed by univariate logistic regression analysis. The indices with $\mathrm{P}<0.05$ were selected and introduced into multivariate logistic regression analysis to identify the independent risk factors for $\mathrm{CR}$. According to receiver operating characteristic (ROC) curve analysis, the predictive value of blood cell parameterrelated indicators for $\mathrm{CR}$ was determined. An area under the curve (AUC) $>0.70$ was regarded as good predictive value. $\mathrm{P}<0.05$ was considered to be statistically significant.

Based on the propensity score matching (PSM) method, the clinical baseline data of the two groups were balanced, and the regression model variables included age, sex, previous diabetes mellitus (DM), hypertension, stroke, smoking, alcohol consumption and systolic blood pressure (SBP), diastolic blood pressure (DBP) and heart rate of the patients after admission. The propensity score of each patient was calculated by the 1:1 nearest matching method, and caliper matching was employed to limit the logarithmic standard deviation of the propensity score to 0.10 to prevent the difference between each pair of matched individuals.

\section{Results}

\section{Baseline Patient Characteristics}

Among the 436 patients with AMI, the average age of the CR group was $70.20 \pm 9.98$ years old and that of the non-CR group was $62.35 \pm 12.08$ years old. This difference was statistically significant $(\mathrm{P}<0.001)$. In the comparisons between clinical characteristics, the SBP, HR, angiotensin-converting enzyme inhibitor (ACEI)/angiotensin-receptor blocker $(\mathrm{ARB})$ and $\beta$-blocker treatment differed between $\mathrm{CR}$ patients and controls $(\mathrm{P}<0.001)$. The patients with $\mathrm{CR}$ had a significantly higher percentage of females than the patients in the non-CR group $(\mathrm{P}=0.008)$. In addition, the proportion of patients with emergency percutaneous coronary intervention (PCI) in the non-CR group was higher than that in the $\mathrm{CR}$ group, and the difference was statistically significant $(\mathrm{P}<0.001)$. Moreover, the results showed that patients in the $\mathrm{CR}$ group had a lower DBP and less statin treatment than those in the non-CR group (both $\mathrm{P}<0.05$ ). Regarding previous $\mathrm{DM}$, hypertension, stroke, smoking, drinking and culprit vessels, there were no statistically significant differences between the two groups (all $\mathrm{P}>0.05$ ). Through evaluation by the PSM method, 90 pairs of patients in the two groups were successfully matched. There were no significant differences in the variables after matching, except for emergency PCI and ACEI/ARB treatment (Table 1).

The blood cell parameters of the study participants in the two groups after PSM are shown in Table 2. There were no significant differences between the two groups regarding the levels of MCV, MCH, MCHC, RDW, PLT, MPV, PCT, LY, $\mathrm{NE}$, or BA (all $\mathrm{P}>0.05$ ). The WBC, PDW, MO, MLR, MHR and NLR values were higher in the CR group than in the non-CR group $(\mathrm{P}<0.05)$. In contrast, compared with the $\mathrm{CR}$ group, the $\mathrm{RBC}, \mathrm{HGB}, \mathrm{HCT}$ and $\mathrm{EO}$ levels were significantly higher in the non-CR group $(\mathrm{P}<0.05)$.

\section{The Results of Logistic Regression Analysis After PSM}

According to the results of univariable logistic regression analysis after PSM, WBC (OR=1.08, 95\% CI: 1.01-1.16, $\mathrm{P}=0.035), \mathrm{RBC}(\mathrm{OR}=0.52,95 \% \mathrm{CI}: 0.32-0.85, \mathrm{P}=0.008)$, MHR ( $\mathrm{OR}=2.32,95 \% \mathrm{CI}: 1.58-3.42, \mathrm{P}<0.001)$, MLR $(\mathrm{OR}=6.64,95 \%$ CI: $2.69-16.43, \mathrm{P}<0.001)$ and emergency $\mathrm{PCI}(\mathrm{OR}=0.46,95 \%$ CI: $0.25-0.84, \mathrm{P}=0.011)$ were risk factors for CR (Table 3). Moreover, the MLR (OR=3.57, 95\% CI: 1.28-9.97, $\mathrm{P}=0.015)$ and MHR (OR=1.80, 95\% CI: $1.02-3.20$, $\mathrm{P}=0.043$ ) levels were independent risk factors for $\mathrm{CR}$ in multivariate logistic regression analysis (Table 3 ).

\section{The Results of ROC Curve Analysis}

In the ROC curve analysis, the effective cut-off point of the MLR was at least 0.61 to predict CR with $64 \%$ sensitivity and $87 \%$ specificity ( $\mathrm{AUC}=0.80,95 \% \mathrm{CI}: 0.73-0.87, \mathrm{P}<0.001$ ) before PSM (Figure $1 \mathrm{~A}$ ). In addition, a meaningful MLR result was also obtained after PSM ( $\mathrm{AUC}=0.74,95 \% \mathrm{CI}$ : $0.65-0.83, \mathrm{P}<0.001$ ), with $63 \%$ sensitivity and $80 \%$ specificity, and the cut-off was also 0.61 (Figure 1B). Before and after PSM, the AUCs of MHR were 0.78 and 0.73, respectively, 
Table I Comparison of Baseline Data Between Two Groups Before and After PSM

\begin{tabular}{|c|c|c|c|c|c|c|}
\hline \multirow[t]{2}{*}{ Factors } & \multicolumn{3}{|c|}{ Before PSM } & \multicolumn{3}{|c|}{ After PSM } \\
\hline & Non-CR $(n=327)$ & $C R(n=109)$ & P-value & Non-CR $(n=90)$ & $C R(n=90)$ & P-value \\
\hline Age, years & $62.35 \pm 12.08$ & $70.20 \pm 9.98$ & $<0.001$ & $71.03 \pm 11.42$ & $70.01 \pm 10.09$ & 0.525 \\
\hline Women, $\mathrm{n}(\%)$ & 93 (28.4\%) & $46(42.2 \%)$ & 0.008 & 35 (38.9\%) & $36(40 \%)$ & 0.879 \\
\hline Hypertension, (\%) & $180(55 \%)$ & $65(59.6 \%)$ & 0.403 & $48(53.3 \%)$ & $51(56.7 \%)$ & 0.653 \\
\hline DM, n (\%) & 77 (23.5\%) & 31 (28.4\%) & 0.305 & $20(22.2 \%)$ & $22(24.4 \%)$ & 0.724 \\
\hline Stroke, n (\%) & $19(5.8 \%)$ & $9(8.3 \%)$ & 0.367 & $3(3.3 \%)$ & $5(5.6 \%)$ & 0.718 \\
\hline Smoker, n (\%) & $143(43.7 \%)$ & $47(43.1 \%)$ & 0.911 & $37(41.1 \%)$ & $39(43.3 \%)$ & 0.763 \\
\hline Drinker, n (\%) & $75(22.9 \%)$ & $22(20.2 \%)$ & 0.550 & $13(14.4 \%)$ & $19(21.1 \%)$ & 0.242 \\
\hline $\mathrm{SBP}, \mathrm{mmHg}$ & $131.32 \pm 22.91$ & $118.06 \pm 24.78$ & $<0.001$ & $122.83 \pm 22.93$ & $119.98 \pm 24.88$ & 0.424 \\
\hline $\mathrm{DBP}, \mathrm{mmHg}$ & $73(65,82)$ & $70(60.5,77.5)$ & 0.014 & $71(63,78)$ & $70(62,77)$ & 0.910 \\
\hline $\mathrm{HR}$, beat $/ \mathrm{min}$ & $80(68,91)$ & $88.5(75,109.5)$ & $<0.001$ & $88(75,98)$ & $86(72,105)$ & 0.882 \\
\hline ACEI/ARB treatment, $n(\%)$ & $160(48.9 \%)$ & $16(14.7 \%)$ & $<0.001$ & $34(37.8 \%)$ & $13(14.4 \%)$ & $<0.001$ \\
\hline$\beta$-blocker treatment, $n$ (\%) & 191 (58.4\%) & $42(38.5 \%)$ & $<0.001$ & $45(50 \%)$ & $37(41.1 \%)$ & 0.231 \\
\hline Statin treatment, n (\%) & 321 (98.2\%) & $102(93.6 \%)$ & 0.035 & 87 (96.7\%) & $84(93.3 \%)$ & 0.494 \\
\hline Culprit vessels & & & 0.236 & & & 0.747 \\
\hline LAD, n (\%) & $168(51.4 \%)$ & $35(32.1 \%)$ & & $40(44.4 \%)$ & $29(32.2 \%)$ & \\
\hline LCX, n (\%) & $44(13.5 \%)$ & $14(12.8 \%)$ & & $15(16.7 \%)$ & $14(15.6 \%)$ & \\
\hline RCA, n (\%) & $89(27.2 \%)$ & $14(12.8 \%)$ & & $19(21.1 \%)$ & $12(13.3 \%)$ & \\
\hline Emergency PCl, n (\%) & $17 \mid(52.3 \%)$ & 38 (34.9\%) & 0.002 & 49 (54.4\%) & $32(35.6 \%)$ & 0.011 \\
\hline
\end{tabular}

Abbreviations: PSM, propensity score matching; DM, diabetes mellitus; SBP, systolic blood pressure; DBP, diastolic blood pressure; HR, heart rate; ACEI/ARB, angiotensinconverting enzyme inhibitor/angiotensin-receptor blocker; PCI, percutaneous coronary intervention; LAD, left anterior descending; LCX, left circumflex artery; RCA, right coronary artery.

Table 2 Comparison of Blood Cell Parameters Between Two Groups After PSM

\begin{tabular}{|l|l|l|l|}
\hline Variables & Non-CR $(\mathbf{n}=90)$ & CR $(\mathbf{n = 9 0})$ & P-value \\
\hline $\mathrm{WBC}, \times 10^{9} / \mathrm{L}$ & $9.66(7.71,12.95)$ & $11.13(9.28,14.09)$ & 0.009 \\
$\mathrm{RBC}, \times 10^{12} / \mathrm{L}$ & $4.25 \pm 0.64$ & $3.98 \pm 0.65$ & 0.007 \\
$\mathrm{HGB}, \mathrm{g} / \mathrm{L}$ & $128.67 \pm 19.91$ & $122.57 \pm 20.96$ & 0.047 \\
$\mathrm{HCT}, \mathrm{L} / \mathrm{L}$ & $0.39 \pm 0.06$ & $0.37 \pm 0.06$ & 0.035 \\
$\mathrm{MCV}, \mathrm{fL}$ & $91.34 \pm 5.47$ & $93 \pm 6.56$ & 0.066 \\
$\mathrm{MCH}, \mathrm{pg}$ & $30.35 \pm 2.27$ & $30.87 \pm 2.56$ & 0.155 \\
$\mathrm{MCHC}, \mathrm{g} / \mathrm{L}$ & $332.08 \pm 13.88$ & $331.92 \pm 13.24$ & 0.939 \\
$\mathrm{RDW}, \%$ & $13.43 \pm 1.48$ & $13.46 \pm 1.31$ & 0.902 \\
$\mathrm{PLT}, \times 10^{9} / \mathrm{L}$ & $203.2 \pm 66.81$ & $217.61 \pm 81.55$ & 0.196 \\
$\mathrm{MPV}, \mathrm{fL}$ & $11.14 \pm 1.24$ & $10.73 \pm 1.65$ & 0.063 \\
$\mathrm{PCT}, \%$ & $0.23 \pm 0.06$ & $0.23 \pm 0.08$ & 0.699 \\
$\mathrm{PDW}, \%$ & $13.4(12,16)$ & $15.7(12.7,16.35)$ & 0.015 \\
$\mathrm{LY}, \times 10^{9} / \mathrm{L}$ & $1.33(0.84,1.82)$ & $1.16(0.73,1.50)$ & 0.126 \\
$\mathrm{MO}, \times 10^{9} / \mathrm{L}$ & $0.45(0.34,0.74)$ & $0.8(0.52,1.05)$ & $<0.001$ \\
$\mathrm{NE}, \times 10^{9} / \mathrm{L}$ & $7.83(5.92,10.55)$ & $8.77(6.95,11.99)$ & 0.053 \\
$\mathrm{EO}, \times 10^{9} / \mathrm{L}$ & $0.03(0.01,0.06)$ & $0(0,0.03)$ & 0.003 \\
$\mathrm{BA}, \times 10^{9} / \mathrm{L}$ & $0.02(0.01,0.03)$ & $0.01(0,0.03)$ & 0.313 \\
$\mathrm{MLR}$ & $0.41(0.25,0.58)$ & $0.75(0.44,1.45)$ & $<0.00 \mathrm{I}$ \\
$\mathrm{MHR}, \times 10^{9} / \mathrm{L}$ & $1.24(0.86,1.94)$ & $2.20(1.33,3.21)$ & $<0.00 \mathrm{I}$ \\
$\mathrm{NLR}$ & $6.42(3.93,10.89)$ & $9.25(5.11,13.5 \mathrm{I})$ & 0.025 \\
\hline
\end{tabular}

Abbreviations: PSM, propensity score matching; WBC, white blood cell count; RBC, red blood cell count; HGB, haemoglobin; HCT, haematocrit; MCV, mean corpuscular volume; $\mathrm{MCH}$, mean corpuscular haemoglobin; $\mathrm{MCHC}$, mean corpuscular haemoglobin concentration; RDW, red blood cell distribution width; PLT, platelet count; MPV, mean platelet volume; PCT, plateletcrit; PDW, platelet distribution width; LY, lymphocyte count; MO, monocyte count; NE, neutrophil count; EO, eosinophil count; BA, basophil count; MLR, monocyte-to-lymphocyte ratio; MHR, monocyte-to-haematocrit ratio; NLR, neutrophil-to-lymphocyte ratio. 
Table 3 Predictors of CR in Multivariable Logistic Regression Analysis After PSM

\begin{tabular}{|l|l|l|l|l|}
\hline \multirow{2}{*}{ Variables } & \multicolumn{2}{|c|}{ Univariable Analysis } & \multicolumn{2}{c|}{ Multivariable Analysis } \\
\cline { 2 - 5 } & OR (95\% Cl) & P-value & OR (95\% CI) & P-value \\
\hline WBC & $1.08(1.01-1.16)$ & 0.035 & $0.93(0.83-1.05)$ & 0.228 \\
RBC & $0.52(0.32-0.85)$ & 0.008 & $0.79(0.41-1.54)$ & 0.494 \\
PDW & $1.01(0.95-1.07)$ & 0.843 & - & - \\
MHR & $2.32(1.58-3.42)$ & $<0.001$ & $1.80(1.02-3.20)$ & 0.043 \\
MLR & $6.64(2.69-16.43)$ & $<0.001$ & $3.57(1.28-9.97)$ & 0.015 \\
NLR & $1.03(0.99-1.07)$ & 0.108 & $1.02(0.45-2.33)$ & - \\
Emergency PCl & $0.46(0.25-0.84)$ & 0.011 & 0.959 \\
\hline
\end{tabular}

Abbreviations: CR, cardiac rupture; PSM, propensity score matching; WBC, white blood cell count; RBC, red blood cell count; PDW, platelet distribution width; MHR, monocyte-to-haematocrit ratio; MLR, monocyte-to-lymphocyte ratio; NLR, neutrophil-to-lymphocyte ratio; PCI, percutaneous coronary intervention.

which were helpful to distinguish CR patients. In addition, an MHR value $>1.66$ was identified as an effective cut-off point for CR (69\% sensitivity and 76\% specificity) before PSM (Figure 2A). The optimal cut-off of MHR was 2.06 (57\% sensitivity and $81 \%$ specificity) after PSM (Figure 2B).

\section{Discussion}

CR is one of the most serious complications of AMI, and it usually occurs suddenly. Moreover, it is difficult to manage by medical and surgical treatment, and it has a high fatality rate. ${ }^{3}$ We found that among the blood cell parameters, an increase in the MLR and MHR were independently associated with the risk of CR. According to the results of ROC curve analysis, the MLR and MHR were useful to predict CR in patients with AMI.

The MLR and MHR could be new markers that are useful for evaluating CR after combining monocytes and lymphocytes and monocytes and haematocrit, respectively. The use of these parameters combined can provide more cumulative information than each marker alone. Previous studies have shown that a high monocyte count or low lymphocyte count is closely related to adverse cardiovascular events in patients with coronary heart disease. ${ }^{12}$
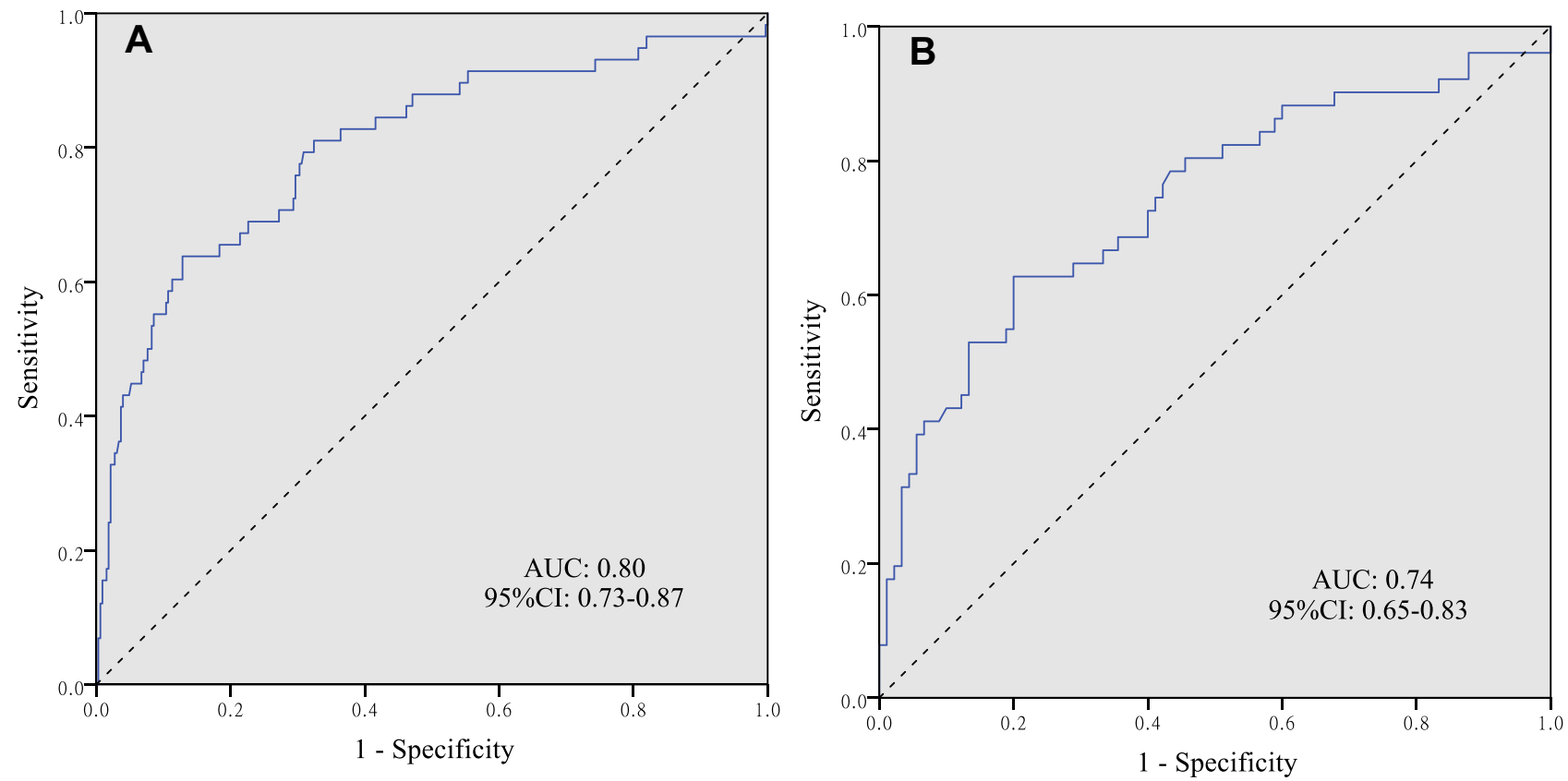

Figure I (A) Receiver operating characteristic (ROC) curve of MLR for predicting CR patients with AMI before PSM. (B) Receiver operating characteristic (ROC) curve of MLR for predicting CR patients with AMI after PSM. 

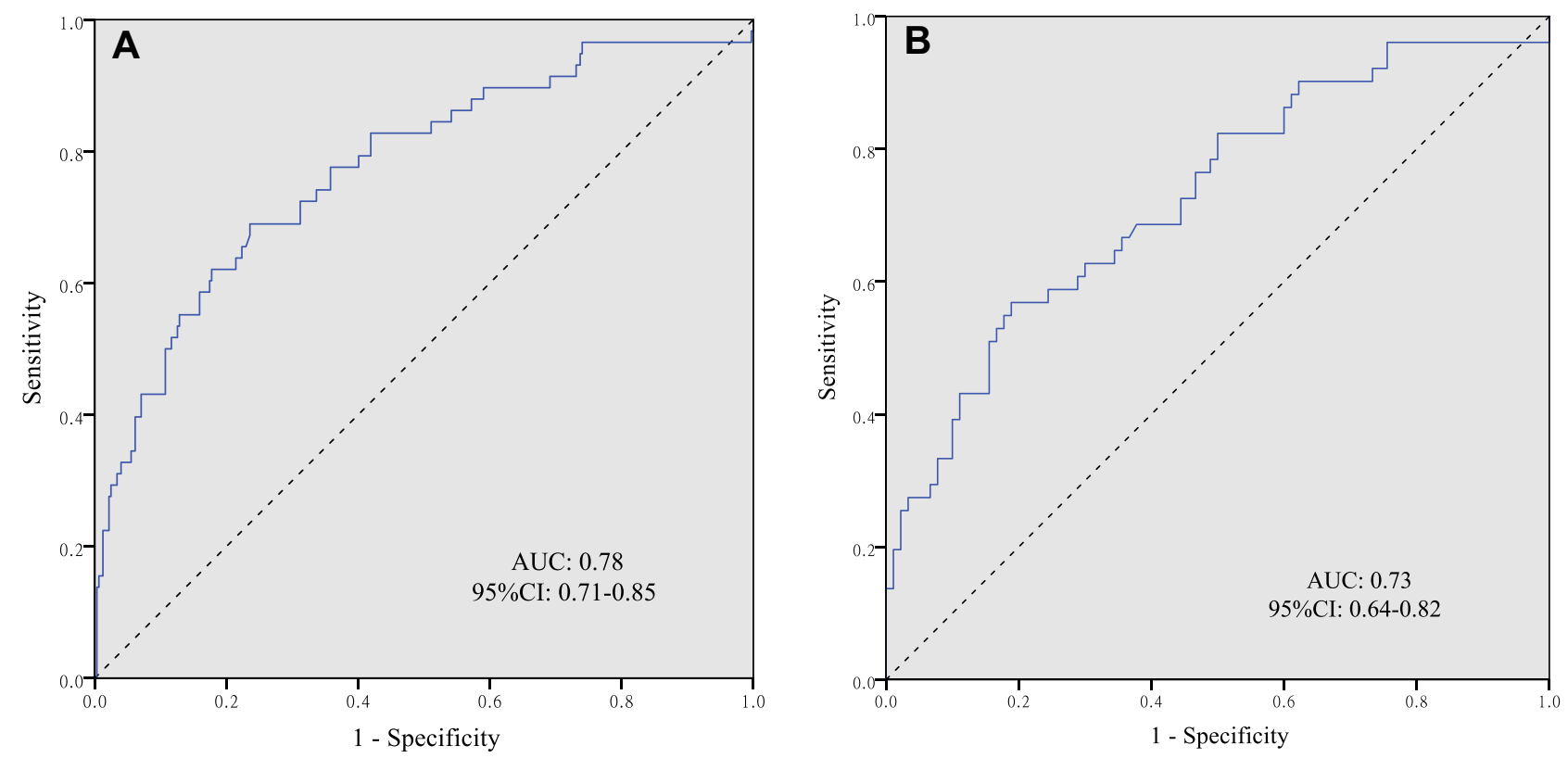

Figure 2 (A) Receiver operating characteristic (ROC) curve of MHR for predicting CR patients with AMI before PSM. (B) Receiver operating characteristic (ROC) curve of MHR for predicting CR patients with AMI after PSM.

Monocytes are the main component of the innate immune system and participate in the process of endogenous inflammation. ${ }^{13}$ Monocytes can migrate from blood to tissue in response to body signals. Then, they can differentiate into inflammatory dendritic cells, macrophages and foam cells, and activate the secretion of proinflammatory cytokines, the production of matrix metalloproteinases and reactive oxidizing substances. This allows for the accumulation of a large number of inflammatory cells that can infiltrate into the infarcted area, which aggravates the damage to myocardial cells in ischaemic area and microcirculation disorders. This then leads to the destruction and dissolution of myocardial cell membrane structure, fibrin cytoskeleton and even cell death. ${ }^{14}$ After ischaemic injury, the combination of TNF- $\alpha$ secreted by macrophages and receptors on the cell surface can amplify an inflammatory cascade in the cardiomyocytes of the infarcted area. This can further lead to the activation of transcription factors that aggravate the fragmentation and degradation of the myocardial collagen fibre structure and can even increase the incidence of CR. ${ }^{15}$ The level of lymphocytes represents cellular immunity and humoral immunity in the body. ${ }^{16}$ In the inflammatory state, the induction and adhesion of chemokines can lead to a large amount of lymphocyte infiltration, and effector $\mathrm{T}$ cells are recruited into infarcts to release proinflammatory transmitters. The immune system also produces a group of regulatory $\mathrm{T}$ lymphocytes with anti-inflammatory effects. ${ }^{17}$ Therefore, the main function of lymphocytes is to inhibit the inflammatory response of the body. Gary found that the MLR increased the risk of severe limb ischaemia and other vascular events in peripheral arterial occlusive disease. ${ }^{18}$ A recent study has shown that the MLR is associated with adverse hospital outcomes in patients with ST-elevation myocardial infarction (STEMI) undergoing PCI. ${ }^{19}$ Ji's research showed that the MLR can predict the severity of CAD through the SYNTAX score. ${ }^{20}$ Fan et al reported that a high MLR in AMI patients treated with PCI was independently related to the risk of 6-month death. ${ }^{6}$ In a study of 306 patients, Wang found that the MLR was an independent predictor of major adverse cardiovascular events in STEMI patients. ${ }^{7}$ Patients with low haematocrit levels might be at a high risk of decreased oxygen supply, which could aggravate myocardial necrosis. Toss et $\mathrm{al}^{21}$ reported that haematocrit was associated with the risk of myocardial infarction in young Swedish men. Kosiborod's study showed that among anaemic patients with heart failure after myocardial infarction, a decrease in haematocrit is associated with a marked decline in health status. ${ }^{22}$ In addition, emergency PCI is treated as an effective measure to prevent $\mathrm{CR}$ by reducing the area of myocardial infarction at present. ${ }^{23-25}$ In this study, the number of patients with emergency PCI in the non-CR group was markedly higher than that in the CR group. Moreover, in univariable logistic regression analysis, emergency PCI was a protective factor for CR. This study took the lead in exploring the relationship between the MLR, 
MHR and CR. The results indicated that the MLR and MHR were independently related to the risks of CR and could be used as effective predictors. Therefore, the MLR and MHR could be useful as conventional, efficient, rapid and inexpensive biomarkers, and they may provide new insights for the screening and treatment of high-risk CR patients.

This study is a retrospective analysis, which has bias in statistics. In addition, due to the small sample size, it is impossible to analyse the types of cardiac rupture in subgroups. Thus, further in-depth and high-quality studies are needed. This study illustrates that the results obtained for the MLR and MHR indicate a possible relationship between inflammatory parameters and CR. It may provide a theoretical reference for front-line clinicians to identify high-risk CR patients early. Furthermore, in addition to the revascularization of culprit arteries as preventive measure, it is available to control inflammation in the development of CR in the future.

\section{Ethical Approval}

This research was an anonymous retrospective study, which was approved by the ethics committees of the First Affiliated Hospital of Nanchang University and Jiangxi Provincial People's Hospital Affiliated to Nanchang University. This study was conducted in accordance with the Declaration of Helsinki. And informed consent was waived by the ethics committees due to the retrospective nature of the study. We confirm that the privacy of the participants was preserved, and the data was anonymized and maintained with confidentiality. The patient data accessed complied with relevant data protection and privacy regulations.

\section{Acknowledgments}

The authors would like to thank all the staff members at the First Affiliated Hospital of Nanchang University and Jiangxi Provincial People's Hospital Affiliated to Nanchang University. Kai Dai, Zhibing Li, Yafei Luo, Qianhui Xiong, Yao Xiong, and Zhifang Song are co-first authors for this study.

\section{Funding}

This study was supported by the National Natural Science Foundation of China (82000234) and partially supported by the Jiangxi Province Natural Science Foundation (20202BABL206004).

\section{Disclosure}

The authors declared that there were no potential conflicts of interest with the research, authorship, and publication of this article.

\section{References}

1. Figueras J, Alcalde O, Barrabés JA, et al. Changes in hospital mortality rates in 425 patients with acute ST-elevation myocardial infarction and cardiac rupture over a 30-year period. Circulation. 2008;118(25):2783-2789. doi:10.1161/CIRCULATIONAHA.108.776690

2. Sulzgruber P, El- Hamid F, Koller L, et al. Long-term outcome and risk prediction in patients suffering acute myocardial infarction complicated by post- infarction cardiac rupture. Int J Cardiol. 2017;227:399-403. doi:10.1016/j.ijcard.2016.11.037

3. Lopez-Sendon J, Gurfinkel EP, Lopez de Sa E, et al. Factors related to heart rupture in acute coronary syndromes in the global registry of acute coronary events. Eur Heart J. 2010;31(12):1449-1456. doi:10.1093/eurheartj/ehq061

4. Qian G, Wu C, Chen YD, et al. Predictive factors of cardiac rupture in patients with ST-elevation myocardial infarction. J Zhejiang Univ Sci B. 2014;15(12):1048-1054. doi:10.1631/jzus.B1400095

5. Qian G, Jin RJ, Fu ZH, et al. Development and validation of clinical risk score to predict the cardiac rupture in patients with STEMI. Am J Emerg Med. 2017;35(4):589-593. doi:10.1016/j.ajem.2016.12.033

6. Fan Z, Ji H, Li Y, et al. Prognostic utility of the combination of monocyte-to-lymphocyte ratio and neutrophil-to-lymphocyte ratio in patients with NSTEMI after primary percutaneous coronary intervention: a retrospective cohort study. BM J Open. 2018;8(10):e023459. doi:10.1136/bmjopen2018-023459

7. Wang Q, Ma J, Jiang Z, et al. Association of lymphocyte-to-monocyte ratio with in-hospital and long-term major adverse cardiac and cerebrovascular events in patients with ST-elevated myocardial infarction. Medicine. 2017;96:e7897. doi:10.1097/MD.0000000000007897

8. Thygesen K, Alpert JS, Jaffe AS, et al. Fourth universal definition of myocardial infarction (2018). J Am Coll Cardiol. 2018;72(18):2231-2264. doi:10.1016/j.jacc.2018.08.1038

9. Bakris G, Ali W, Parati G. ACC/AHA versus ESC/ESH on hypertension guidelines: JACC guideline comparison. J Am Coll Cardiol. 2019;73 (23):3018-3026. doi:10.1016/j.jacc.2019.03.507

10. Mühlhauser I, Meyer G. Evidence base in guideline generation in diabetes. Diabetologia. 2013;56(6):1201-1209. doi:10.1007/s00125-013-2872-6 
11. Tian L, Yang Y, Zhu J, et al. Impact of previous stroke on short-term myocardial reinfarction in patients with acute ST segment elevation myocardial infarction: an observational multicenter study. Medicine (Baltimore). 2016;95(6):e2742. doi:10.1097/MD.0000000000002742

12. Ley K, Miller YI, Hedrick CC, et al. Monocyte and mac rophage dynam ics during atherogenesis. Arterioscler Thromb Vasc Biol. 2011;31(7):15061516. doi:10.1161/ATVBAHA.110.221127

13. Creemers EE, Cleutjens JP, Smits JF, et al. Matrix metalloproteinase inhibition after myocardial infarction: a new approach to prevent heart failure? Circ Res. 2001;89(3):201-210. doi:10.1161/hh1501.094396

14. Solomon SD, Pfeffer MA. Renin-angiotensin system and cardiac rupture after myocardial infarction. Circulation. 2002;106(17):2167-2169. doi:10.1161/01.cir.0000034039.01213.39

15. Askari T, Brennanan L, Zhou X, et al. Myeloperoxidase and plasminogen activ ator inhibitor I play a central role in ventricular remodeling after myocardial in farction. Exp Med. 2003;197(5):615-624. doi:10.1084/jem.20021426

16. Tanaka M, Masuda S, Matsuo Y, et al. Hyperglycemia and inflammatory property of circulating monocytes are associated with inflammatory property of carotid plaques in patients undergoing carotid endarterectomy. J Atheroscler Thromb. 2016;23(10):1212-1221. doi:10.5551/jat.32680

17. Bansal SS, Ismahil MA, Goel M, et al. Dysfunctional and proinflammatory regulatory T-lymphocytes are essential for adverse cardiac remodeling in ischemic cardiomyopathy. Circulation. 2019;139(2):206-221. doi:10.1161/CIRCULATIONAHA.118.036065

18. Gary T, Pichler M, Belaj K, et al. Lymphocyte-to-monocyte ratio: a novel marker for critical limb ischemia in PAOD patients. Int $J$ Clin Pract. 2014;68(12):1483-1487. doi:10.1111/ijcp.12495

19. Kurtul A, Yarlioglues M, Celik IE, et al. Association of lymphocyte- to- monocyte ratio with the no- reflow phenomenon in patients who underwent a primary percutaneous coronary intervention for ST- elevation myocardial infarction. Coron Artery Dis. 2015;26(8):706-712. doi:10.1097/ MCA.0000000000000301

20. Ji H, Li Y, Fan Z, et al. Monocyte/lymphocyte ratio predicts the severity of coronary artery disease: a syntax score assessment. BMC Cardiovasc Disord. 2017;17(1):90. doi:10.1186/s12872-017-0507-4

21. Toss F, Nordström A, Nordström P. Association between hematocrit in late adolescence and subsequent myocardial infarction in Swedish men. Int $J$ Cardiol. 2013;168(4):3588-3593. doi:10.1016/j.ijcard.2013.05.065

22. Kosiborod M, Krumholz HM, Jones PG, et al. The relationship between anemia, change in hematocrit over time and change in health status in patients with heart failure after myocardial infarction. J Card Fail. 2008;14(1):27-34. doi:10.1016/j.cardfail.2007.09.009

23. Honda S, Asaumi Y, Yamane T, et al. Trends in the clinical and pathological characteristics of cardiac rupture in patients with acute myocardial infarction over 35 years. J Am Heart Assoc. 2014;3:e00984. doi:10.1161/JAHA.114.000984

24. French JK, Hellkamp AS, Armstrong PW, et al. Mechanical complications after percutaneous coronary intervention in ST-elevation myocardial infarction (from APEX-AMI). Am J Cardiol. 2010;105:59-63. doi:10.1016/j.amjcard.2009.08.653

25. Gong W, Feng S, Wang X, et al. Beta-blockers reduced the risk of cardiac rupture in patients with acute myocardial infarction: a meta-analysis of randomized control trials. Int $J$ Cardiol. 2017;232:171-175. doi:10.1016/j.ijcard.2017.01.035

Risk Management and Healthcare Policy

\section{Publish your work in this journal}

Risk Management and Healthcare Policy is an international, peer-reviewed, open access journal focusing on all aspects of public health, policy, and preventative measures to promote good health and improve morbidity and mortality in the population. The journal welcomes submitted papers covering original research, basic science, clinical \& epidemiological studies, reviews and evaluations, guidelines, expert opinion and commentary, case reports and extended reports. The manuscript management system is completely online and includes a very quick and fair peer-review system, which is all easy to use. Visit http://www.dovepress.com/testimonials.php to read real quotes from published authors.

Submit your manuscript here: https://www.dovepress.com/risk-management-and-healthcare-policy-journal 